\title{
Extending holomorphic maps in infinite dimensions
}

\author{
by Bui DaC TAC (Hanoi)
}

\begin{abstract}
Studying the sequential completeness of the space of germs of Banachvalued holomorphic functions at a points of a metric vector space some theorems on extension of holomorphic maps on Riemann domains over topological vector spaces with values in some locally convex analytic spaces are proved. Moreover, the extendability of holomorphic maps with values in complete $C$-spaces to the envelope of holomorphy for the class of bounded holomorphic functions is also established. These results are known in some special cases.
\end{abstract}

Introduction. In finite-dimensional complex analysis the extension problem for holomorphic maps has been investigated by many authors: Ivashkovich [7], Kobayashi [10], Shiffman [16], ... In recent years some authors have considered this problem in the locally convex infinite-dimensional case, for example Hirschowitz [6], Schottenloher [14], ...

The aim of this paper is to study the extension of holomorphic maps on Riemann domains over topological vector spaces with values in some locally convex analytic spaces. We make use of the definition and properties of locally convex analytic spaces as in [12]. Some of our results are known in the finite-dimensional case.

The concept of an $L$-space and of a locally convex analytic space having the finite-dimensional extension property are given in Section 1. In this section we prove that every holomorphic map from a Riemann domain over an $L$-space into a locally convex analytic space having the finite-dimensional extension property can be extended to the envelope of holomorphy. Some examples of locally convex analytic spaces having the finite-dimensional extension property are also given in Section 1.

The extension of holomorphic maps with values in the domain of existence of a Banach-valued holomorphic function is considered in Section 2. Studying the sequential completeness of the space of germs of Banach-valued holomorphic functions at a point of a metric vector space we prove that ev-

1991 Mathematics Subject Classification: Primary 32D10. 
ery holomorphic map from a Riemann domain over a metric vector space into the domain of existence of a Banach-valued holomorphic function satisfying some additional condition can be holomorphically extended to the envelope. Using the obtained result we give an extension of holomorphic maps with values in sequentially holomorphically convex Riemann domains. In a special case, this result has been proved by Schottenloher [14].

Finally, in Section 3 we prove that every holomorphic map from a Riemann domain over a topological vector space into a finite-dimensional complete $C$-space can be extended to a holomorphic map on the envelope of holomorphy for the class of bounded holomorphic functions. In the 1-dimensional case the result has been proved by Sibony [17] and by Hirschowitz [6] for the general case.

We wish to express our gratitude to Dr. N. V. Khue for his guidance and help.

$\S 1$. Extending holomorphic maps with values in locally convex analytic spaces having the finite-dimensional extension property

1.1. Definition. We say that a locally convex analytic space $X$ has the finite dimensional extension property if every holomorphic map on a finite-dimensional Riemann domain over a Stein manifold with values in $X$ can be holomorphically extended to the envelope of holomorphy.

1.2. Definition. A topological vector space $E$ is called an $L$-space if every pseudoconvex Riemann domain over $E$ is the domain of existence of a holomorphic function.

\subsection{Some examples}

a) Every Lindelöf locally convex space having a Schauder decomposition is an $L$-space [1].

b) Every metric vector space with a translation invariant metric $d$ and with a Schauder decomposition such that $x \mapsto \log d(x, 0)$ is plurisubharmonic is an $L$-space [2].

c) $l^{\infty}(A)$ with $A$ an uncountable set is not an $L$-space [8].

1.4. Theorem. Let $\Omega$ be a Riemann domain over an L-space E. Then every holomorphic map on $\Omega$ with values in a locally convex analytic space having the finite-dimensional extension property can be extended to a holomorphic map on the envelope of holomorphy $\widehat{\Omega}$ of $\Omega$.

To prove the theorem we need the following, which is known in the locally convex case [13]. 
1.5. Proposition. Let $\Omega$ be a Riemann domain over a topological vector space $E$ defined by a locally homeomorphic map $p: \Omega \rightarrow E$ and let $\Omega$ be a domain of holomorphy. Then $\Omega$ is pseudoconvex.

Proof. Given a compact set $K$ in $\Omega$, take an invariant continuous pseudometric $\rho$ on $E$ such that $\delta=d_{\rho}(K)>0$, where

$$
d_{\rho}(K)=\inf \left\{d_{\rho}(x): x \in K\right\}
$$

and $d_{\rho}(x)$ denotes the boundary pseudodistance function induced by $\rho$,

$d_{\rho}(x)=\sup \{r>0$ : there exists a neighbourhood $U$ of $x$ such that

$p \mid U: U \rightarrow B_{\rho}(p x, r):=\{z \in E: \rho(z, p x)<r\}$ is a homeomorphism $\}$.

Let $x \in \widehat{K}_{\Omega}:=\left\{x \in \Omega:|f(x)| \leq \sup _{K}|f(y)| \forall f \in H(\Omega)\right\}$, where $H(\Omega)$ denotes the space of holomorphic functions on $\Omega$ equipped with the compactopen topology. For each $f \in H(\Omega)$, consider the Taylor expansion of $f$ at $x$,

$$
f(x+h)=\sum_{n=0}^{\infty} l_{n}(x, h)
$$

where

$$
l_{n}(x, h)=\frac{1}{2 \pi i} \int_{|\lambda|=1} \frac{f(x+\lambda h) d \lambda}{\lambda^{n+1}}, \quad h \in B_{\rho}(0, \delta) .
$$

We prove that (1) is uniformly convergent on $B_{\rho}\left(0, \delta_{1}\right)$ with $\delta_{1}=\delta / 2$. Let $y \in B_{\rho}\left(0, \delta_{1}\right)$. Then

$$
K y:=K+\{2 \lambda y:|\lambda| \leq 1\} \subset \Omega .
$$

Take a balanced neighbourhood $W$ of zero in $E$ such that

$$
M=\sup \{|f(z+2 \lambda y+\omega)|: z \in K, \omega \in W,|\lambda| \leq 1\}<\infty .
$$

Then

$$
\begin{aligned}
\left|l_{n}(x, 2 y+\omega)\right| & \leq \sup _{K}\left|l_{n}(z, 2 y+\omega)\right| \\
& \leq \sup _{K}\left|\frac{1}{2 \pi i} \int_{|\lambda|=1} \frac{f(z+\lambda(2 y+\omega)) d \lambda}{\lambda^{n+1}}\right| \leq M \quad \forall n \geq 0 .
\end{aligned}
$$

Hence

$$
\sum_{n \geq 0} \sup _{W}\left|l_{n}(x, y+\omega / 2)\right|=\sum_{n \geq 0} \sup _{W}\left|l_{n}\left(x, \frac{1}{2}(2 y+\omega)\right)\right| \leq \sum_{n \geq 0} M / 2^{n}<\infty .
$$

Thus every $f \in H(\Omega)$ can be extended to a holomorphic function on $x+$ $B_{\rho}\left(0, \delta_{1}\right)$. This yields $d_{\rho}\left(\widehat{K}_{\Omega}\right)>0$.

Now we assume that $F$ is an arbitrary finite-dimensional subspace of $E$ and $K$ a compact set in $\Omega_{F}:=p^{-1}(F)$. Since $\widehat{K}_{\Omega_{F}} \subseteq \widehat{K}_{\Omega}$ and $d_{\rho \mid F} \geq d_{\rho} \mid \Omega_{F}$, it follows that $d_{\rho \mid F}\left(\widehat{K}_{\Omega_{F}}\right) \geq d_{\rho}\left(\widehat{K}_{\Omega}\right)>0$. Since $\rho$ can be chosen such 
that $\rho \mid F$ defines the topology of $F$ we infer that $\Omega_{F}$ is pseudoconvex. The proposition is proved.

Proof of Theorem 1.4. Let $f$ be a given holomorphic function on $\Omega$ with values in $X$. By $\mathcal{S}_{X}$ we denote the sheaf of germs of holomorphic maps on $\widehat{\Omega}$ with values in $X$. Consider $\mathcal{S}_{X}^{f}$, the envelope of holomorphy of $f$. Then $\mathcal{S}_{X}^{f}$ is the connected component of $\mathcal{S}_{X}$ containing $\left\{f_{x}: x \in \Omega\right\}$ and $f$ has a canonical extension $\widehat{f}: \mathcal{S}_{X}^{f} \rightarrow X$. Since $\left(\mathcal{S}_{X}^{f}, \widetilde{p}\right)$, where $\widetilde{p}:=\widehat{p} \circ \pi, \widehat{p}: \widehat{\Omega} \rightarrow E$, $\pi: \mathcal{S}_{X}^{f} \rightarrow \widehat{\Omega}$ are canonical maps, is a Riemann domain over $E$ such that every holomorphic function on $\Omega$ can be extended to a holomorphic function on $\mathcal{S}_{X}^{f}$ and since $E$ is an $L$-space, to prove the theorem it suffices to show that $\mathcal{S}_{X}^{f}$ is pseudoconvex.

Let $F$ be finite-dimensional subspace of $E$. To prove the pseudoconvexity of $\widetilde{p}^{-1}(F)$, by a theorem of Docquier-Grauert [4] it remains to show that $\widetilde{p}^{-1}(F)$ is Hartogs convex.

Let $\sigma: H_{k}(\varepsilon) \rightarrow \widetilde{p}^{-1}(F)$ be a holomorphic embedding, where $H_{k}(\varepsilon)$ denotes the $k$-dimensional Hartogs diagram consisting of all points $z=$ $\left(z_{1}, \ldots, z_{k}\right) \in D^{k}, D=\{z \in \mathbb{C}:|z|<1\}$, such that

$$
\max \left\{\left|z_{1}\right|, \ldots\left|z_{k-1}\right|<\varepsilon\right\} \quad \text { or } \quad\left|z_{k}\right|>1-\varepsilon .
$$

Consider the holomorphic map $\pi \sigma: H_{k}(\varepsilon) \rightarrow \widehat{p}^{-1}(F)$. Since $\widehat{\Omega}$ is pseudoconvex and $\operatorname{dim} F<\infty$, it follows that $\widehat{p}^{-1}(F)$ is Stein. Hence $\pi \sigma$ can be extended to a holomorphic map $\widehat{\pi \sigma}: D^{k} \rightarrow \widehat{p}^{-1}(F)$. Set $\tau=\widehat{\pi \sigma}$. It is easy to see that $\tau$ is locally biholomorphic. By hypothesis $\widehat{f} \sigma: H_{k}(\varepsilon) \rightarrow X$ can be extended to a holomorphic map $f$ on $D^{k}$. Define a holomorphic map $\widehat{\sigma}: D^{k} \rightarrow X$ by

$$
\widehat{\sigma}(z)=\left[\widehat{f}\left(\tau \mid V_{z}\right)^{-1}\right]_{\tau(z)}
$$

where $V_{z}$ is a neighbourhood of $z$ such that $\tau \mid V_{z}$ is biholomorphic. Then $\widehat{\sigma}$ is a holomorphic extension of $\sigma$. Thus $\widetilde{p}^{-1}(F)$ is Hartogs convex. The proof of the theorem is complete.

\subsection{Some examples}

1. Every Banach-Lie group has the finite-dimensional extension property.

Proof. Let $G$ be a Banach-Lie group. To prove that $G$ has the finitedimensional extension property it suffices to show that $G$ has the Hartogs extension property, i.e. every holomorphic map from $H_{k}(r)$ into $G$ can be extended to a holomorphic map on $D^{k}$.

Let $f: H_{k}(r) \rightarrow G$ be a holomorphic map. Consider the topological group $H\left(H_{k}(r), G\right)$ consisting of holomorphic maps of $H_{k}(r)$ into $G$ equipped with the compact-open topology and the exponential map exp :TG $\rightarrow G$, where $T G_{e}$ denotes the tangent space to $G$ at the unit element $e$ of $G$. 
Note that exp is biholomorphic on a neighbourhood $V$ of zero in $T G_{e}$. This implies that the map

$$
\widehat{\exp }: H\left(H_{k}(r), T G_{e}\right) \rightarrow H\left(H_{k}(r), G\right)
$$

is biholomorphic on the neighbourhood $W$ of zero in $H\left(H_{k}(r), T G_{e}\right)$, where

$$
W=\left\{g \in H\left(H_{k}(r), T G_{e}\right): g\left(H_{k}(s)\right) \subset V\right\}
$$

with $0<s<r$.

Since $\widehat{\exp }(W)$ generates $H\left(H_{k}(r), G\right)$, there exist $g_{1}, \ldots, g_{p} \in W$ such that $f=\widehat{\exp } g_{1} \ldots \widehat{\exp } g_{p}$. Let $\widehat{g}_{1}, \ldots, \widehat{g}_{p}$ be holomorphic extensions of $g_{1}, \ldots, g_{p}$ to $D^{k}$. Then $\widehat{\exp } \widehat{g}_{1} \ldots \widehat{\exp \widehat{g}_{p}}$ is a holomorphic extension of $f$ to $D^{k}$.

R e m a r k. In case $\operatorname{dim} G<\infty$, the finite-dimensional extension property of $G$ has been proved in [1].

2. Let $X$ be a locally convex analytic space which is modelled by analytic sets in open sets of a locally convex sequentially complete space. Let $X$ satisfy the weak disc condition. Then $X$ has the finite-dimensional extension property.

Here we say that $X$ satisfies the weak disc condition if the canonical map $H(D, X) \rightarrow H\left(D^{*}, X\right)$, where $D^{*}=D \backslash\{0\}$, is an embedding.

Proof. It suffices to show that every holomorphic map $f: H_{k}(r) \rightarrow X$ can be extended to a holomorphic map on $D^{k}$. Let $\mathcal{S}_{X}^{f}$ be the envelope of holomorphy of $f$. We shall prove that $\mathcal{S}_{X}^{f} \cong D^{k}$. For this it suffices to check that $\mathcal{S}_{X}^{f}$ satisfies the weak disc condition [16].

Let $\left\{\sigma_{n}\right\} \subset H\left(D, \mathcal{S}_{X}^{f}\right)$ converge to $\sigma$ in $H\left(D^{*}, \mathcal{S}_{X}^{f}\right)$. By hypothesis $\left\{\widehat{f} \sigma_{n}\right\}$, where $\widehat{f}: \mathcal{S}_{X}^{f} \rightarrow X$ is the canonical extension of $f$, converges to $g$ in $H(D, X)$. Take a neighbourhood $U$ of $g(0)$ in $X$ such that $U$ is isomorphic to an analytic set in an open subset of a locally convex sequentially complete space. Then there exist $0<\varepsilon<1$ and $N$ such that

$$
\widehat{f} \sigma_{n}\left(D_{\varepsilon}\right) \subset U \quad \forall n \geq N, D_{\varepsilon}=\varepsilon D .
$$

Put $K=\overline{\operatorname{conv} \bigcup_{n \geq N} \widehat{f} \sigma_{n}\left(D_{\varepsilon / 2}\right)}$. Let $B$ be the Banach space spanned by $K$, equipped with the norm induced by $K$. Then $\widehat{f} \sigma_{n}: \partial D_{\varepsilon / 2} \rightarrow B$ is continuous for every $n \geq N$. Hence from the relation

$$
\widehat{f} \sigma_{n}(z)=\frac{1}{2 \pi i} \int_{|\lambda|=\varepsilon / 2} \frac{f \sigma_{n}(\lambda) d \lambda}{\lambda-z}
$$

it follows that $\widehat{f} \sigma_{n}: D_{\varepsilon / 2} \rightarrow B$ is holomorphic for every $n \geq N$. Consider for each $n \geq N$ the map $\widetilde{\sigma}_{n}: D_{\varepsilon / 2} \rightarrow \lim _{W \ni 0} H(W, B)$ defined by

$$
\tilde{\sigma}_{n}(\lambda)=\left[\sigma_{n}(\lambda) \circ \theta_{\hat{e} \sigma_{n}(\lambda)}\right]_{0}
$$


where $\theta_{u}(\tau)=u+\tau$ and $\widehat{e}: \mathcal{S}_{X}^{f} \rightarrow D^{k}$ is an extension of the canonical embedding $e: H_{k}(\varepsilon) \rightarrow D^{k}$. Then $\widetilde{\sigma}_{n} \rightarrow \widetilde{\sigma}$ in $H\left(D_{\varepsilon / 2} \backslash\{0\}, \lim H(W, B)\right)$, hence in $H\left(D_{\varepsilon / 2}, \lim H(W, B)\right)$. Since $\left\{\widetilde{\sigma}_{n}\right\}$ is bounded in $\lim H(W, B)$ there exists a neighbourhood $W$ of zero in $\mathbb{C}^{k}$ such that $\widetilde{\sigma}_{n}(\lambda) \in H(W, B)$ for $|\lambda| \leq \varepsilon / 2$ and $n \geq N$. Obviously $\sigma$ can be holomorphically extended to $0 \in D$ by setting $\sigma(0)=\widetilde{\sigma}(0) \theta_{\hat{e} \sigma(0)}^{-1}$. It remains to show that $\sigma_{n} \rightarrow \sigma$ in $H\left(D, \mathcal{S}_{X}^{f}\right)$. Consider the neighbourhood $\widetilde{W}$ of $\sigma\left(\partial D_{\varepsilon / 2}\right)$ given by

$$
\widetilde{W}=\bigcup\left\{\left(\widehat{e} \sigma(\lambda)+x,[\sigma(\lambda)]_{\hat{e} \sigma(\lambda)+x}\right):|\lambda|=\varepsilon / 2, x \in W\right\} .
$$

Then there exists $N^{\prime} \geq N$ such that

$$
\sigma_{n}\left(\partial D_{\varepsilon / 2}\right) \subset \widetilde{W} \quad \text { for } n \geq N^{\prime} .
$$

For each $|\lambda|=\varepsilon / 2$ and $n \geq N^{\prime}$ there exists a neighbourhood $U(n, \lambda)$ of $\widehat{e} \sigma(\lambda)$ in $\widehat{e} \sigma(\lambda)+W$ such that

$$
\sigma_{n}(\lambda) x=\sigma(\lambda) x \quad \text { for all } x \in U(n, \lambda) .
$$

Thus

$$
\sigma_{n}(\lambda) x=\sigma(\lambda) x \quad \text { for every } x \in \widehat{e} \sigma(\lambda)+W \text { and }|\lambda|=\varepsilon / 2,
$$

and hence also for every $x \in \hat{e} \sigma(\lambda)+W$ and $\lambda \in D_{\varepsilon / 2}$. This means that $\left\{\sigma_{n}\right\}$ converges to $\sigma$ in $H\left(D, \mathcal{S}_{X}^{f}\right)$.

Remark. When $\operatorname{dim} X<\infty$, the result has been proved by Shiffman [16]. Our proof is different.

§2. Extending holomorphic maps with values in the domain of existence of a Banach-valued holomorphic function. In this section we investigate the extension of holomorphic maps with values in the domain of existence of a Banach-valued holomorphic function. A result of Hirschowitz [5] yields the existence of such a domain which is not the domain of existence of a $\mathbb{C}$-valued holomorphic function.

Before stating the main result of this section (Theorem 2.6) we prove Proposition 2.3, which will be used in the proof of Theorem 2.6. We give the following two definitions.

2.1. Definition. A topological vector space $E$ is called an $H Z$-space if every hypoholomorphic function on a connected open subset $\Omega$ of $E$ which is continuous at some point of $\Omega$, is holomorphic on $\Omega$.

Here we recall that a map $f$ from an open set $\Omega$ in a topological vector space $E$ into a locally convex space $F$ is hypoholomorphic if $f$ is Gateauxholomorphic and continuous on every compact set in $\Omega$.

2.2. Definition. Let $f$ be a holomorphic function on a Riemann domain $(X, p)$ over a locally convex space $F$ with values in a Banach space $B$. We say 
that $f$ satisfies the countable condition if there exists an increasing sequence $\left\{\alpha_{j}\right\}$ of continuous seminorms on $F$ such that $\widetilde{f}$ given by

$$
\tilde{f}(z)=\left[f\left(p \mid V_{z}\right)^{-1} \circ \theta_{p(z)}\right]_{0}
$$

is a holomorphic map from $X$ into $\lim _{U \in \mathcal{U}} H^{\infty}(U, B)$, where $\mathcal{U}$ is a neighbourhood basis of zero in $\varliminf_{\text {im } F / \operatorname{Ker}} \alpha_{j}$.

2.3. Proposition. Let $\Omega$ be a Riemann domain over an $H Z$-space and $F$ a sequentially complete locally convex space. Then every holomorphic map of $\Omega$ into $F$ can be extended to a holomorphic map on $\widehat{\Omega}$.

Proof. Let $f: \Omega \rightarrow F$ be a holomorphic map. Consider the holomorphic composition map

$$
\bar{f}: \Omega \stackrel{f}{\rightarrow} F \rightarrow \prod\left\{\mathbb{C}: l \in F^{\prime}\right\} .
$$

Then $\bar{f}$ can be extended to a holomorphic map

$$
\widetilde{\bar{f}}: \widehat{\Omega} \rightarrow \prod\left\{\mathbb{C}: l \in F^{\prime}\right\} .
$$

Put

$$
\begin{aligned}
& \Omega_{1}=\operatorname{Int} \widetilde{\bar{f}}^{-1}(F), \\
& V_{x}(f)=\left\{h \in E: \sum \alpha\left(\widetilde{\bar{f}}^{(k)}(x, h)\right)<\infty\right. \\
&\text { for every continuous seminorm } \alpha \text { on } F\}, \\
& \stackrel{\circ}{V}_{x}(f)=\operatorname{Int}\left\{y \in V_{x}(f): \sum_{\text {hypoholomorphic at } y\} .}^{\widetilde{\bar{f}}^{(k)}(x, h) \text { is }}\right.
\end{aligned}
$$

We need the following two lemmas.

2.4. LEMMA. Let $x \in \Omega_{1}$ and let $U$ be a balanced neighbourhood of zero in $E$ such that

$$
x+U \subset \bigcap\left\{\stackrel{\circ}{V}_{x}(l \circ f): l \in F^{\prime}\right\} .
$$

Then $x+\delta U \subset \stackrel{\circ}{V}_{x}(f), \forall 0 \leq \delta<1$.

Proof. Let $K$ be a compact set in $U$ and $\alpha$ a continuous seminorm on $F$. Since for every $l \in(F / \operatorname{Ker} \alpha)^{\prime}$ and $h \in K, \lim _{k \rightarrow \infty} l \circ \widetilde{\bar{f}}^{(k)}(x, h)=0$, by the Banach-Steinhaus theorem we have

$$
\begin{aligned}
& M(\alpha, K)=\sup \left\{\alpha\left(\widetilde{\widetilde{f}}^{(k)}(x, h)\right): k \geq 0, h \in K\right\} \\
& \quad=\sup \left\{l \circ \widetilde{\bar{f}}^{(k)}(x, h): k \geq 0, h \in K, l \in(F / \operatorname{Ker} \alpha)^{\prime},\|l\| \leq 1\right\}<\infty .
\end{aligned}
$$

Hence for all $0 \leq \delta<1$ and $h \in \delta K$ we have

$$
\sum \alpha\left(\widetilde{\widetilde{f}}^{(k)}(x, h)\right) \leq \sum \delta^{k} M(\alpha, K)<\infty .
$$


Since $F$ is sequentially complete, this yields that $x+\delta U \subset \stackrel{\circ}{V}_{x}(\widetilde{\bar{f}})$ for all $0 \leq \delta<1$.

2.5. LemmA. Let $\left\{x_{\alpha}\right\} \subset \Omega_{1}, x_{\alpha} \rightarrow x \in \partial \Omega_{1}$ and $U$ a balanced neighbourhood of zero in $E$ such that

$$
x+U \subset \bigcap\left\{\stackrel{\circ}{V}_{x}(l \circ \widetilde{\bar{f}}): l \in F^{\prime}\right\} .
$$

Then there exists a balanced neighbourhood $V$ of zero in $E$ such that $x_{\alpha}+V \subset$ ${\stackrel{\circ}{V_{x_{\alpha}}}}(\widetilde{\bar{f}})$ for sufficiently large $\alpha$.

Pr o of. Take a balanced neighbourhood $W$ of zero in $E$ such that $W+$ $W \subseteq U$. Then for $\alpha$ sufficiently large we have $x_{\alpha}+W \subseteq x_{\alpha}+W+W \subseteq x+U$. Since $x_{\alpha}+W$ is balanced at $x_{\alpha}$ and $l \circ f$ is hypoholomorphic on $x_{\alpha}+W$ we have $x_{\alpha}+W \subseteq \stackrel{\circ}{V}_{x_{\alpha}}(l \circ \widetilde{\bar{f}})$ for all $l \in F^{\prime}$. From Lemma 2.4 it follows that $x_{\alpha}+V \subseteq \stackrel{\circ}{V}_{x_{\alpha}}(\widetilde{\bar{f}})$, where $V=W / 2$. The lemma is proved.

To prove Proposition 2.3, it remains to apply Lemma 2.5. Indeed, take a balanced neighbourhood $U$ of zero in $E$ such that

$$
x+U \subseteq \bigcap\left\{V_{x}(l \circ \widetilde{\bar{f}}): l \in F^{\prime}\right\} .
$$

By Lemma 2.5 we have $\left.x_{\alpha}+V \subseteq{\stackrel{\circ}{V_{x}}}_{(\widetilde{\bar{f}}}\right)$ for sufficiently large $\alpha$ and for some balanced neighbourhood $V$ of zero in $E$. Take a balanced neighbourhood $W$ of zero in $E$ such that $W+W \subseteq V$ and $x-x_{\alpha} \in W$ for sufficiently large $\alpha$. We have for every $h \in W$

$$
x+h=x+x_{\alpha}-x_{\alpha}+h \in x_{\alpha}+W+W \subseteq x_{\alpha}+V \subseteq \stackrel{\circ}{V}_{x_{\alpha}}(\widetilde{\bar{f}}) .
$$

Thus $\widetilde{\bar{f}}$ is hypoholomorphically extended to $x$. Hence $\Omega_{1}$ is closed. Since without loss of generality we may assume that $\widehat{\Omega}$ is connected it follows that $\Omega_{1}=\widehat{\Omega}$. Hence, by hypothesis we infer that $\widetilde{\bar{f}}$ is holomorphic on $\widehat{\Omega}$.

2.6. TheOREM. Let $\Omega$ be a Riemann domain over an $H Z$-space and let $(X, p)$ be the domain of existence of a Banach-valued holomorphic function $h$ satisfying the countable condition. Then every holomorphic map from $\Omega$ into $X$ extends to a holomorphic map on $\widehat{\Omega}$.

We need the following

2.7. LEMMA. Let $E$ be a metric vector space and $B$ a Banach space. Then $\lim \left\{H^{\infty}(U, B): U \ni 0 \in E\right\}$ is regular and sequentially complete.

Pr o of. Take a set $T$ such that $B$ is isomorphic to a subspace of $l^{\infty}(T)$.

a) First we show that $\lim H^{\infty}\left(U, l^{\infty}(T)\right)$ is regular. Observe that $H^{\infty}\left(U, l^{\infty}(T)\right) \cong l^{\infty}\left(T, H^{\infty}(U)\right)$ for every neighbourhood $U$ of zero in $E$. 
Hence

$$
\lim H^{\infty}\left(U, l^{\infty}(T)\right) \cong \lim l^{\infty}\left(T, H^{\infty}(U)\right) .
$$

Since the space $\prod_{T} H(U)$ is Montel and the canonical maps

$$
l^{\infty}(T, H(U)) \rightarrow \prod_{T} H(U) \rightarrow \prod_{T} H(V)
$$

are continuous for all neighbourhoods $U, V$ of zero in $E$ such that $V \subseteq U$, it follows that $S\left(l^{\infty}\left(T, H^{\infty}(U)\right)\right.$, the closed unit ball in $l^{\infty}\left(T, H^{\infty}(U)\right)$, is closed in $l^{\infty}\left(T, H^{\infty}(V)\right)$ for all neighbourhoods $U, V$ of zero in $E$ with $V \subseteq U$. Hence $\lim H^{\infty}\left(U, l^{\infty}(T)\right)$ is regular [2].

Assume now that $A$ is a bounded set in $\lim H^{\infty}(U, B)$. Then there exists a neighbourhood $U$ of zero in $E$ such that $A$ is bounded in $H^{\infty}\left(U, l^{\infty}(T)\right)$. Hence $A$ is bounded in $H^{\infty}(U, B)$.

b) Assume that $\left\{g_{k}\right\}$ is a Cauchy sequence in $\lim H^{\infty}(U, B)$. By a), [lim $\left.H^{\infty}(U, B)\right]^{\prime} \cong \lim ^{\infty}(U, B)^{\prime}$ is a Fréchet space and there exists a neighbourhood $W$ of zero in $E$ such that $\left\{g_{k}\right\}$ is bounded in $H^{\infty}(W, B)$. This implies that $\left\{g_{k}\right\}$ is convergent to $g \in\left[\lim H^{\infty}(U, B)\right]^{\prime \prime}$. We prove that $g \in \varliminf^{\infty} H^{\infty}(U, B)$ and hence $g_{k} \rightarrow g$ in $\varliminf^{\infty}(U, B)$. Note that $g_{k} \rightarrow g$ in $[\lim H(U, B)]^{\prime \prime}$. On the other hand, since $g_{k}(t) \rightarrow g(t)$ in $H(W)$ for every $t \in T$ and

$$
\begin{aligned}
\sup \{|g(t)(x)|: t \in T, & x \in W\} \\
& \leq \sup \left\{\left|g_{k}(t)(x)\right|: t \in T, x \in W, k \geq 1\right\}<\infty
\end{aligned}
$$

it follows that $g \in l^{\infty}\left(T, H^{\infty}(W)\right) \cong H^{\infty}\left(W, l^{\infty}(T)\right)$. Since $g_{k}(x) \in B$, $\forall k \geq 1, \forall x \in W$, it follows that $g \in H^{\infty}(W, B)$.

The lemma is proved.

Proof of Theorem 2.6. Let $f: \Omega \rightarrow X$ be a holomorphic map. By Proposition 2.3, $p \circ f$ can be extended to a holomorphic map $g$ on $\widehat{\Omega}$. Consider the holomorphic map

$$
\tilde{f}: \Omega \rightarrow \lim \left\{H^{\infty}(U, B): U \in \mathcal{U}\right\},
$$

where $\mathcal{U}$ is a neighbourhood basis of zero in $\varliminf \widehat{F / K} \operatorname{er} \alpha_{j}$, defined by

$$
\widetilde{f}(z)=\left[h\left(p \mid V_{f(z)}\right)^{-1} \circ \theta_{g(z)}\right]_{0}
$$

where $V_{f(z)}$ is a neighbourhood of $f(z)$ such that $p: V_{f(z)} \cong p\left(V_{f(z)}\right)$ and $\theta_{g(z)}(\tau)=\tau+g(z)$. By Lemma 2.7 and Proposition 2.3, $\widetilde{f}$ can be extended to a holomorphic map $\widehat{\widetilde{f}}: \widehat{\Omega} \rightarrow \lim \left\{H^{\infty}(U, B): U \in \mathcal{U}\right\}$.

Let $z_{0} \in \partial \Omega$. Take a sequence $\left\{z_{n}\right\} \subset \Omega, z_{n} \rightarrow z_{0}$. Since $\left\{\widehat{\widetilde{f}}\left(z_{n}\right)\right\}$ is bounded in $\lim \left\{H^{\infty}(U, B): U \in \mathcal{U}\right\}$, we can find a neighbourhood $W$ of

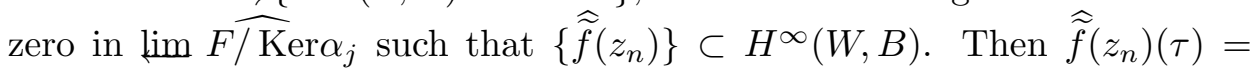
$h\left(p \mid V_{f\left(z_{n}\right)}\right)^{-1}\left(\tau+g\left(z_{0}\right)\right)$ for $\tau$ belonging to a neighbourhood of zero in $W$. 
This implies that $h\left(p \mid V_{f\left(z_{n}\right)}\right)^{-1}$ extends holomorphically to $W+g\left(z_{n}\right)$ for every $n \geq 1$. Thus $h$ is extended holomorphically to $g\left(z_{0}\right)+W / 2$ to the function $\widehat{\widetilde{f}}\left(z_{0}\right) \circ \theta_{g\left(z_{0}\right)}^{-1}$. Hence we can define a holomorphic map $\widehat{f}: \widehat{\Omega} \rightarrow X$ by

$$
\widehat{f}(z)=\left(g(z),\left[\widehat{\widetilde{f}}(z) \circ \theta_{g(z)}^{-1}\right]_{g(z)}\right) \quad \text { for } z \in \widehat{\Omega},
$$

which is a holomorphic extension of $f$.

The theorem is proved.

Let $(Z, q)$ be a Riemann domain over a locally convex space $F$. We recall the following two definitions.

2.8. Definition [14]. A sequence $\left\{z_{n}\right\} \subset Z$ will be called a boundary sequence relative to $q$ if $\left\{z_{n}\right\}$ has no accumulation point in $Z$ and $\left\{q z_{n}\right\}$ is convergent to a point $z^{*}$ in $F$ and if for every neighborhood $V$ of $z^{*}$ there exists $N$ such that the remainder $\left\{z_{n}\right\}_{n \geq N}$ is contained in a connected component of $q^{-1}(V)$.

2.9. Definition [14]. The Riemann domain $Z$ is called sequentially holomorphically convex if for every boundary sequence $\left\{z_{n}\right\}$ relative to any locally biholomorphic map $p: Z \rightarrow F$ there exists $g \in H(X)$ such that $\sup \left|g\left(z_{n}\right)\right|=\infty$.

2.10. TheOREM. Let $(X, p)$ be a sequentially holomorphically convex Riemann domain over a locally convex space $F$ such that every element of $H(X)$ satisfies the countable property and let $\Omega$ be a Riemann domain over an $H Z$-space $E$. Then every holomorphic map of $\Omega$ into $X$ can be extended to a holomorphic map on $\widehat{\Omega}$.

P r o of. For each finite subset $I$ of $H(X)$, let $\left(X_{I}, p_{I}\right)$ denote the envelope of $I$ over $F$. Put

$$
\widetilde{X}=\bigcap\left\{X_{I}: I \subset H(X), \text { card } I<\infty\right\} .
$$

Consider the canonical map $\theta: X \rightarrow \widetilde{X}$. Let $X_{1}=\theta(X)$ and $p_{1}=p_{I} \mid X_{1}$. Note that $\left(X_{1}, p_{1}\right)$ is a Riemann domain over $F$. By the relation $H(X) \cong$ $H\left(X_{1}\right)$, it follows that $(X, p) \cong\left(X_{1}, p_{1}\right)$. Let $f: \Omega \rightarrow X$ be a holomorphic map. By Theorem 2.6 there exists a map $\widehat{f}: \widehat{\Omega} \rightarrow \widetilde{X}$ such that $\left.\widehat{f}\right|_{\Omega}=f$ and $\widehat{f}: \widehat{\Omega} \rightarrow X_{I}$ is holomorphic for every finite subset $I$ of $H(X)$. Put $Z=\widehat{f}^{-1}\left(X_{1}\right)$. To complete the proof, it remains to check that $Z$ is closed in $\widehat{\Omega}$. Let $z_{0} \in \partial Z$ and let $\rho$ be an invariant continuous pseudometric on $E$ such that $\delta=d_{\rho}\left(z_{0}\right)>0$. Let $z_{1} \in S:=\left\{y \in \widehat{\Omega}: p y \in B_{\rho}\left(p z_{0}, \delta\right)\right\}$. Define a map $k:[0,1] \rightarrow \widehat{\Omega}$ by

$$
k(t)=(p \mid B)^{-1}\left(p z_{0}+t\left(p z_{1}-p z_{0}\right)\right) .
$$


Put

$$
T=\{t \in[0,1]: k(t) \in \widehat{\Omega} \backslash Z\}, \quad s=\sup T .
$$

Since $T$ is closed in $[0,1]$ and $\widehat{\Omega} \backslash Z$ is closed in $\widehat{\Omega}$, it follows that $s \in T$. Assume $s<1$. Then there exists a sequence $\left\{t_{n}\right\}$ such that $t_{n} \searrow s$. We prove that $\left\{z_{n}\right\}:=\left\{\widehat{f} k\left(t_{n}\right)\right\}$ is a boundary sequence in $X$ relative to an arbitrary locally biholomorphic map $\pi: X \rightarrow F$. Obviously $\left\{z_{n}\right\}$ has no accumulation point in $X$ and $\left\{\pi z_{n}\right\}=\left\{\pi \widehat{f} k\left(t_{n}\right)\right\} \rightarrow \pi \widehat{f} k(s)$ in $F$. Given a neighbourhood $V$ of $\pi \widehat{f} k(s)$, take $\varepsilon>0$ such that $\widehat{f} k(s, s+\varepsilon) \subseteq \pi^{-1}(V)$. Since $\widehat{f} k(s, s+\varepsilon)$ is connected a remainder of $\left\{z_{n}\right\}$ is contained in a connected component of $\pi^{-1}(V)$. Thus $\left\{z_{n}\right\}$ is a boundary sequence of $X$. By hypothesis, there exists $g \in H(X)$ such that $\sup \left|g\left(z_{n}\right)\right|=\infty$. This contradicts the fact that $g\left(z_{n}\right)=g \widehat{f} k\left(t_{n}\right) \rightarrow(g \widehat{f} k)(s)$. Thus $s=1$, i.e. $z_{1}=k(1) \in \Omega \backslash Z$. Since $z_{1}$ is arbitrary, we have $S \subset \widehat{\Omega} \backslash Z$, i.e. $\widehat{\Omega} \backslash Z$ is open.

Theorem 2.10 is proved.

\subsection{Some examples}

1) Every holomorphic function on a Riemann domain over either a metric vector space or a Lindelöf topological vector space satisfies the countable property.

2) Every holomorphic function on an $\omega$-domain satisfies the countable property.

Here, as in [14], by an $\omega$-domain we mean a Riemann domain $X$ over a locally convex space $F$ such that there exists a countable open cover $\left\{U_{j}\right\}$ of $X$ having the following property:

For each $j$ there exists a continuous seminorm $\alpha_{j}$ on $F$ and $s>0$ such that $d_{\alpha_{j}}\left(U_{j}\right)>s$ and $\sup \left\{|f(y)|: \alpha_{j}(x-y)<s\right\}<\infty$.

From the definition of an $\omega$-domain it follows that every $f \in H(X)$ satisfies the countable property.

3) It is easy to see that every holomorphic function on a Riemann domain over a locally convex space which is a surjective limit of Fréchet spaces satisfies the countable property.

$\S 3$. Extending holomorphic maps with values in a $C$-space. Let $X$ be a locally convex analytic space and let $C_{X}$ denote the Carathéodory pseudometric on $X$ defined by

$$
C_{X}(x, y)=\sup \left\{\rho_{\Delta}(f(x), f(y)): f \in H(X, D)\right\}
$$

where $\rho_{\Delta}$ denotes the Bergman-Poincaré metric on $D$. As in [17] we say that $X$ is a $C$-space if $C_{X}$ is a metric on $X$. Moreover, if $X$ is complete for $C_{X}$ then $X$ is called a complete $C$-space. In the finite-dimensional case, it 
is known [17] that the original topology of every $C$-space $X$ is induced by $C_{X}$ when $X$ is a complete $C$-space.

In this section we prove the following

3.1. Theorem. Let $X$ be a complete finite-dimensional $C$-space and $\Omega$ a Riemann domain over a topological vector space $E$. Then every holomorphic map from $\Omega$ into $X$ can be extended to a holomorphic map on $\widehat{\Omega}_{\infty}$, the envelope of holomorphy of $\Omega$ for $H^{\infty}(\Omega)$.

Proof. a) Let $f: \Omega \rightarrow B$ be a bounded holomorphic map, where $B$ is a Banach space. For each $x^{\prime} \in B^{\prime}$ we denote by $\widehat{x^{\prime} f}$ the holomorphic extension of $x^{\prime} f$ to $\widehat{\Omega}_{\infty}$. Define

$$
[\widehat{f}(z)]\left(x^{\prime}\right)=\widehat{x^{\prime} f}(z) \quad \text { for } z \in \widehat{\Omega}_{\infty} \text { and } x^{\prime} \in B^{\prime} .
$$

We have

$$
\begin{aligned}
& \sup \left\{\left|\widehat{f}(z)\left(x^{\prime}\right)\right|:\left\|x^{\prime}\right\| \leq 1\right\}=\sup \left\{\left|\widehat{x^{\prime} f}(z)\right|:\left\|x^{\prime}\right\| \leq 1\right\} \\
& \leq C \sup \left\{\left|x^{\prime} f(y)\right|:\left\|x^{\prime}\right\| \leq 1, y \in \Omega\right\} \quad \text { for some constant } C .
\end{aligned}
$$

Hence $\widehat{f}(z) \in B^{\prime \prime}$. Moreover, $\widehat{f}$ is a bounded holomorphic function with values in $B^{\prime \prime}$. Since $f(\Omega) \subseteq B$, it follows that $\widehat{f}\left(\widehat{\Omega}_{\infty}\right) \subseteq B$.

b) Let $\delta: X \rightarrow H^{\infty}(X)^{\prime}$ be the canonical map. Since $X$ is a complete $C$-space, it follows that $\delta$ is a holomorphic homeomorphism of $X$ on $\delta(X)$. Thus $\delta(X)$ is an analytic set in $H^{\infty}(X)^{\prime}$ of finite dimension [12]. By a) we infer that $\delta f$ can be extended to a holomorphic map $\widehat{\delta f}$. Note that $\widehat{\delta f}\left(\widehat{\Omega}_{\infty}\right) \subseteq \delta(X)$. Since $\delta$ is a holomorphic homeomorphism there exist two analytically rare sets $X^{\prime}$ and $Y^{\prime}$ in $X$ and $\delta(X)$ respectively such that $\delta: X \backslash X^{\prime} \cong \delta(X) \backslash Y^{\prime}$. By induction on $\operatorname{dim} X$, we may assume that $\widehat{\delta f}^{-1}\left(Y^{\prime}\right) \neq \widehat{\Omega}_{\infty}$. Since $\delta^{-1} \widehat{\delta f} \mid \widehat{\Omega}_{\infty} \backslash \widehat{\delta f}^{-1}\left(Y^{\prime}\right)$ is holomorphic and $\delta^{-1} \widehat{\delta f}$ is continuous on $\widehat{\Omega}_{\infty}$ it follows that $\delta^{-1} \widehat{\delta f}$ is a holomorphic extension of $f$.

\section{References}

[1] K. Adachi, M. Suzuki and M. Yoshida, Continuation of holomorphic mappings, with values in a complex Lie group, Pacific J. Math. 47 (1) (1973), 1-4.

[2] A. Bayoumi, The Levi problem and the radius of convergence of holomorphic functions on metric vector spaces, in: Lecture Notes in Math. 843, Springer, 1981, 9-32.

[3] G. Coeuré, Analytic Functions and Manifolds in Infinite Dimensional Spaces, North-Holland Math. Stud. 11, 1974.

[4] F. Docquier und H. Grauert, Levisches Problem und Rungescher Satz für Teilgebiete Steinscher Mannigfaltigkeiten, Math. Ann. 140 (1960), 94-123.

[5] A. Hirschowitz, Prolongement analytique en dimension infinie, Ann. Inst. Fourier (Grenoble) 22 (2) (1972), 255-292.

[6] -, Domaines de Stein et fonctions holomorphes bornées, Math. Ann. 213 (1975), $185-193$. 
[7] S. M. Ivashkovich, Hartogs' phenomenon for holomorphically convex Kähler manifolds, Izv. Akad. Nauk SSSR Ser. Mat. 50 (4) (1986) 866-873.

[8] B. Josefson, A counter-example in the Levi problem, in: Proceedings on Infinite Dimensional Holomorphy, Lecture Notes in Math. 364, Springer, 1974, 168-177.

[9] L. V. Kantorovich and G. P. Akilov, Functional Analysis in Normed Spaces, Gos. Izdat. Fiz.-Mat. Liter., Moscow 1959 (in Russian).

[10] S. Kobayashi, Hyperbolic Manifolds and Holomorphic Mappings, Marcel Dekker, New York 1970.

[11] E. Ligocka and J. Siciak, Weak analytic continuation, Bull. Acad. Pol. Sci. Sér. Sci. Math. Astronom. Phys. 20 (6) (1972), 461-466.

[12] P. Mazet, Analytic Sets in Locally Convex Spaces, North-Holland Math. Stud. 89, 1984.

[13] P. Noverraz, Pseudo-convexité, Convexité Polynomiale et Domaines d'Holomorphie en Dimension Infinie, North-Holland Math. Stud. 3, 1973.

[14] M. Schottenloher, Analytic continuation and regular classes in locally convex Hausdorff spaces, Portugal. Math. 33 (4) (1974), 219-250.

[15] - The Levi problem for domains spread over locally convex spaces, Ann. Inst. Fourier (Grenoble) 26 (1976), 255-292.

[16] B. Shiffman, Extension of holomorphic maps into Hermitian manifolds, Math. Ann. 194 (1971), 249-258.

[17] N. Sibony, Prolongement des fonctions holomorphes bornées et métrique de Carathéodory, Invent. Math. 29 (1975), 205-230.

DEPARTMENT OF MATHEMATICS

PEDAGOGICAL INSTITUTE 1 OF HANOI

HANOI, VIETNAM

Reçu par la Rédaction le 5.11.1988

Révisé le 3.8.1989 